Pacific

Journal of

Mathematics

SOME UNUSUAL COMPARISON PROPERTIES OF CAPILLARY SURFACES

Robert Finn and A.A. Kosmodem'yanskit JR. 


\title{
SOME UNUSUAL COMPARISON PROPERTIES OF CAPILLARY SURFACES
}

\author{
Robert Finn And A.A. Kosmodem’yanskit JR.
}

Under typical physical conditions, the solution of the capillarity equation for a tube of circular section $\mathcal{D}$ will always exceed over $\mathcal{D}$ the solution obtained for a concentric tube of the same material and larger radius. We address here a question raised by $M$. Miranda, as to whether a solution over a general domain $\mathcal{D}_{0}$ will exceed, over that section, the solution over any domain $\mathcal{D}_{1}$ strictly containing $\mathcal{D}_{0}$. We show that whenever a domain $\mathcal{D}_{1}$ admits a zero gravity solution surface in a variational sense for the given contact angle, and has at some point a boundary curvature inward directed and exceeding the ratio of perimeter to area of the section, there is then a subdomain $\mathcal{D}_{0}$ for which a negative answer appears for all sufficiently small gravity $g$; that occurs with height differences inversely proportional to $g$, uniformly over $\mathcal{D}_{0}$.

Under other conditions, positive answers appear. We provide an example in which the limiting behavior as $g \rightarrow 0$ reverses in a discontinuous way, with smooth infinitesimal change of $\partial \mathcal{D}_{0}$. Remarkably, the discontinuous change occurs at a circular cylinder configuration, for which one normally expects stable behavior.

The discussion includes some results that seem to have general geometric interest; notably, we characterize in Theorem 5 all convex domains containing a disk, and for which the ratio of perimeter to area is not less than for the disk.

1.

We are interested in solutions $u(x)$, over a plane domain $\mathcal{D}$, of the capillarity equation

$$
\operatorname{div} T u=B u+2 H, \quad T u \equiv \frac{D u}{\sqrt{1+|D u|^{2}}}
$$

for the height of the free surface interface $\mathcal{S}$ in a vertical capillary tube of section $\mathcal{D}$, positioned in a gravity field. We interpret (1) nondimensionally in terms of the "Bond number" $B=\rho g a^{2} / \sigma$, with $\rho=$ density change across $\mathcal{S}$, $g=$ gravitational acceleration, $\sigma=$ surface tension; here $a$ is a representative length, which is taken as the unit for scaling the position vector $x$ and the 
height $u(x)$. The constant $H$ is to be determined by an eventual volume constraint.

On the boundary $\Sigma=\partial \mathcal{D}$ with exterior normal $\nu$ the transversality condition

$$
\nu \cdot T u=\cos \gamma
$$

is imposed. Geometrically, the surface is required to meet the vertical bounding walls of the tube over $\Sigma$ in a (prescribed) constant angle $\gamma$. We may always suppose $0 \leq \gamma<\pi / 2$; the complementary case $\pi / 2<\gamma \leq \pi$ transforms to that one on replacing $u$ and $H$ by their negatives. If $\gamma=\pi / 2$ then under the conditions we will impose, the unique solution of (1), (2) is the trivial solution $u \equiv$ const.

Our attention will focus on solutions of (1) without volume constraint, in downward directed gravity fields, with the denser fluid below the surface, that is, on solutions of

$$
\operatorname{div} T u=B u
$$

with $B>0$. Solutions of (3) represent the height of the free surface in a vertical capillary tube dipped into an infinite fluid reservoir, with surface height $u=0$ at infinity.

About twenty five years ago Mario Miranda raised in informal conversation the question, as to whether under these conditions a tube of section $\mathcal{D}_{0}$ will raise liquid to a greater height over its section than will a tube of section $D_{1} \supset D_{0}$. In [1] an example is given under which a local singular behavior at a corner point of $\mathcal{D}_{1}$ induces a negative answer to the question relative to a particular subdomain $\mathcal{D}_{0}$, at least over some open subset of $\mathcal{D}_{0}$. Some conditions for a positive answer to the question appear in [4], Sec. 5.3. In Sec. 5.4 of $[4]$ another example is given in which $\mathcal{D}_{1}$ raises a larger volume over $\mathcal{D}_{0}$ than does $\mathcal{D}_{0}$ itself, under conditions for which both solutions are smooth and bounded.

In [10], the latter author re-examined the question in the context of the ratios of the perimeters $p$ to the areas $A$ of the domains, with emphasis on configurations for which $B$ is small. He was led to height relationships depending on the ratios of $p$ to $A$, rather than on inclusion properties of the domains. The present paper pursues that approach further, and develops also other relationships that do derive from geometric inclusion properties; the juxtaposition of results leads to some consequences, notably for the Miranda question, that seem to us remarkable and unexpected.

If $B=0$, Equation (1) becomes

$$
\operatorname{div} T u=2 H .
$$

Under the boundary condition (2) we find from the divergence theorem that $2 H A=p \cos \gamma$. Thus, the value of $H$ cannot be imposed, but is determined 
by the geometry of the base domain. The determination is independent of volume, and a consequence of that is that the shape of the surface interface is the same for every volume.

Every strict solution of (1), (2) in $\mathcal{D}$ satisfies the variational condition

$$
\int_{\mathcal{D}}(\nabla \eta \cdot T u+(B u+2 H) \eta) d x-\oint_{\partial \mathcal{D}} \eta \cos \gamma d s=0
$$

for all $\eta \in \mathcal{Q}(\mathcal{D}) \equiv H^{1,1}(\mathcal{D}) \cap L^{\infty}(\mathcal{D})$. If $B>0$, then under fairly general conditions solutions of (5) will exist, and will in fact be strict solutions of (1), (2) when $\mathcal{D} \in C^{2+\alpha}$, see, e.g., [3], [5], [7], [8], [9], [15], [16]. In the material below we will encounter domains that are only piecewise of that smoothness. For such domains, the existence of solutions of (5) is nevertheless available $[\mathbf{3}, \mathbf{5}, \mathbf{7}]$ when $B>0$, and we will not require further information on boundary smoothness.

As is known, when $B=0$ solutions of (4) (or even of (5)) that are regular in $\mathcal{D}$ do not in general exist under the boundary condition (2); existence can fail even for analytic convex $\mathcal{D}$. Solutions do however exist in many cases of interest; for such configurations, we find:

Theorem 1. Let $u(x)$ be a "variational solution" of (3), (2) in $\mathcal{D}$, in the sense that it is of class $C^{2}$ in $\mathcal{D}$ and that

$$
\int_{\mathcal{D}}(\nabla \eta \cdot T u+B u \eta) d x-\oint_{\partial \mathcal{D}} \eta \cos \gamma=0
$$

for every $\eta \in \mathcal{Q}(\mathcal{D})$, and suppose $B>0$. Suppose there exists a variational solution $U(x)$ of $(4),(2)$ in $\mathcal{D}$, in the sense

$$
\int_{\mathcal{D}}(\nabla \eta \cdot T U+2 H \eta) d x-\oint_{\partial \mathcal{D}} \eta \cos \gamma d s=0
$$

for every $\eta \in \mathcal{Q}(\mathcal{D})$. If $U(x)$ admits lower and upper bounds $m, M$ in $\mathcal{D}$, then

$$
\left|u-\frac{p}{B A} \cos \gamma\right|<M-m
$$

throughout $\mathcal{D}$.

Proof. Choosing $\eta \equiv 1$, we obtain from (7) that $2 H A=p \cos \gamma$, thus determining $H$. From (6) and (7) we find

$$
\int_{\mathcal{D}}(\nabla \eta \cdot(T u-T U)+B(u-U) \eta) d x=\int_{\mathcal{D}}(2 H-B U) \eta d x
$$

for every $\eta \in \mathcal{Q}(\mathcal{D})$. If we replace $U$ in (9) by $U+(2 H / B)-m$, then (9) continues to hold, and the integral on the left will be non-positive for every non-negative $\eta \in \mathcal{Q}(\mathcal{D})$. From Lemma 3 of [7] we conclude $u<$ $U+(2 H / B)-m<(2 H / B)+M-m$. Similarly, we may make that integral non-negative for all such $\eta$ on replacing $U$ by $U+(2 H / B)-M$. There follows $u>U+(2 H / B)-M>(2 H / B)-(M-m)$, as was to be shown. 
This result extends a reasoning of D. Siegel [14] in the case of solutions smooth to the boundary. In interpreting the result, it should be noted that in view of $(3),(p \cos \gamma) / B A$ is exactly the mean value of the solution $u(x)$ over the domain $\mathcal{D}$. Note also that the bound in (8) is independent of $B$, for all positive $B$. We remark that when a bounded variational solution of (4), (2) fails to exist, then the left side of (8) will in general increase without bound, as $B \rightarrow 0$.

In order to apply Theorem 1, one needs criteria for existence and boundedness of variational solutions of (4), (2). For reference, we state here the following necessary and sufficient condition, established in [5] and in [4] Chapter 7 , for existence in a piecewise smooth $\mathcal{D}$ of a "variational" solution of (4) (2), that is smooth interior to $\mathcal{D}$ and assumes the data $\gamma$ in the weak generalized sense imposed by (7).

Property A. A solution $u(x)$ of (5) with $B=0$ exists if and only if, for every subdomain $\mathcal{D}^{*} \subset \mathcal{D}$, such that $\mathcal{D}^{*} \neq \varnothing, \mathcal{D}$ and such that $\mathcal{D}^{*}$ is bounded on $\Sigma$ by subarcs $\Sigma^{*} \subset \Sigma$ and within $\mathcal{D}$ by subarcs $\Gamma^{*}$ of semicircles of radius $A /(p \cos \gamma)$ with the properties:

i) The curvature vector of each $\Gamma^{*}$ is directed exterior to $\mathcal{D}^{*}$, and

ii) each $\Gamma^{*}$ meets $\Sigma$, either in the angle $\gamma$ measured within $\mathcal{D}^{*}$ or else at re-entrant corner points of $\Sigma$,

there holds

$$
\Phi\left(\mathcal{D}^{*} ; \gamma\right)=\left|\Gamma^{*}\right|-\left|\Sigma^{*}\right| \cos \gamma+\frac{p}{A}\left|\mathcal{D}^{*}\right| \cos \gamma>0 .
$$

Every such solution is smooth interior to $\mathcal{D}$, and uniquely determined up to an additive constant.

The utility of (10) derives largely from the circumstance that in configurations typically encountered only a finite number $N$ of $\operatorname{arcs} \Gamma^{*}$ can be found that meet the geometrical requirements, and thus only a finite number of cases need be examined. With reference to our particular needs below, we note that if $\mathcal{D}$ is a rectangle and if $\gamma \geq \pi / 4$, then $N=0$. The requirements are in this case vacuously satisfied, and we conclude that a solution exists. If $\gamma<\pi / 4$, then subdomains bounded as indicated can be found for which $\Phi<0$, and no solution exists.

In conjunction with the above result, one has $([6])$ :

Property B. Suppose that at all "corner" points at which $\partial \mathcal{D}$ consists locally of arcs forming a protruding angle $2 \alpha<\pi$, $\partial \mathcal{D}$ lies locally exterior to a corner consisting of linear segments that meet in angle $2 \alpha$. Then every variational solution of (4), (2) in $\mathcal{D}$ is bounded.

The condition indicated here is chosen for convenience in verification; for a more precise condition, cf. Hypothesis $\alpha(\gamma)$ in [6]. In connection with this property, we note that if $\alpha+\gamma<\pi / 2$ at any corner point, then no variational 
solution of $(4),(2)$ can exist in $\mathcal{D}$. This can be seen by examining (10) for the case in which $\mathcal{D}^{*}$ is the region cut off at the vertex of the corner by a straight segment orthogonal to the angle bisector.

2 .

We are now prepared for applications to Miranda's question. From Theorem 1 follows immediately:

Theorem 2. Let $\mathcal{D}_{0}, \mathcal{D}_{1}$ be plane domains with perimeters $p_{0}, p_{1}$ and areas $A_{0}, A_{1}$, and let $u_{0}(x), u_{1}(x)$ be variational solutions of (3) in the respective domains, corresponding to boundary angle $\gamma$. Suppose there exist variational solutions of (4) in $\mathcal{D}_{0}, \mathcal{D}_{1}$, bounded below and above by $m_{0}, M_{0}$ and by $m_{1}, M_{1}$. Then

$$
\begin{aligned}
& \inf _{\mathcal{D}_{0}} u_{0}-\sup _{\mathcal{D}_{1}} u_{1}>\frac{1}{B}\left(\frac{p_{0}}{A_{0}}-\frac{p_{1}}{A_{1}}\right) \cos \gamma-\left(M_{0}+M_{1}\right)+\left(m_{0}+m_{1}\right) \\
& \inf _{\mathcal{D}_{1}} u_{1}-\sup _{\mathcal{D}_{0}} u_{0}>\frac{1}{B}\left(\frac{p_{1}}{A_{1}}-\frac{p_{0}}{A_{0}}\right) \cos \gamma-\left(M_{0}+M_{1}\right)+\left(m_{0}+m_{1}\right) .
\end{aligned}
$$

Corollary 2.1. Under the hypotheses of Theorem 2, suppose $\mathcal{D}_{0} \subset \mathcal{D}_{1}$. If $\frac{p_{0}}{A_{0}}>\frac{p_{1}}{A_{1}}$ then the Miranda question has a positive answer for all small enough $B$. If $\frac{p_{0}}{A_{0}}<\frac{p_{1}}{A_{1}}$ then the Miranda question has a negative answer for all small enough $B$.

We emphasize that the inclusion relation $\mathcal{D}_{0} \subset \mathcal{D}_{1}$ is not necessary for the inequalities (11), and thus the content of the theorem applies to a much more general class of configurations than were envisaged in Miranda's original question.

It is not difficult to find examples in which the former eventuality of Corollary 2.1 holds. Notably, for any domain $\mathcal{D}_{0}$ that admits a bounded variational solution of (4) and (2), an appropriate $\mathcal{D}_{1} \supset \mathcal{D}_{0}$ can be obtained by similarity transformation. Further, if $\mathcal{D}_{1}$ is a disk then by the isoperimetric inequality any proper subdomain admitting a variational solution of (4) and (2) will serve as $\mathcal{D}_{0}$. Examples for the second eventuality are less evident; however, one has the following general theorem and corollary:

Theorem 3. Let $\mathcal{D}$ be a domain that is piecewise of class $C^{2+\alpha}$, having perimeter $p$ and area A. Suppose there exists a point $q \in \partial \mathcal{D}$ which is either the vertex of a protruding corner or else a smooth point at which the boundary curvature $\kappa$ is directed inward and for which $\kappa(q)>p / A$. Then there exists a subdomain $\mathcal{D}^{\varepsilon} \subset \mathcal{D}$, such that $p^{\varepsilon} / A^{\varepsilon}<p / A$.

Proof. Suppose first that $q$ is a point of boundary smoothness, so that we may describe $\partial \mathcal{D}$ locally in the form

$$
y=\frac{\kappa}{2} x^{2}+o\left(x^{2}\right), \quad \text { with } y^{\prime}=\kappa x+o(x) .
$$




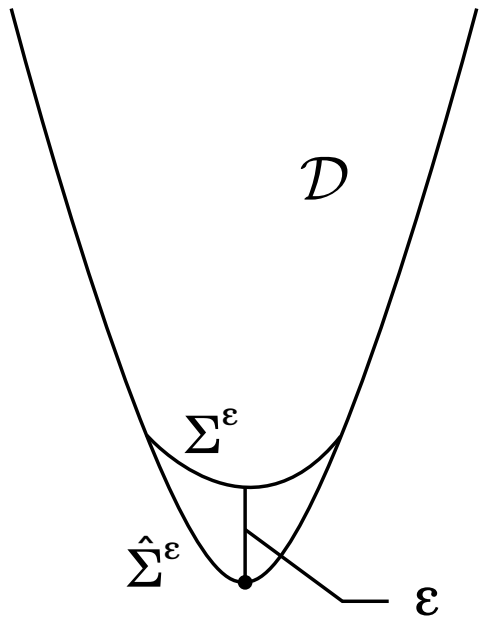

Figure 1. Proof of Theorem 3.

For $\varepsilon>0$ and $\kappa^{\varepsilon}<\kappa$ to be determined, we introduce a new arc $\Sigma^{\varepsilon}$ of the form

$$
y=\varepsilon+\frac{\kappa^{\varepsilon}}{2} x^{2}
$$

as in Figure 1, cutting off an arc $\hat{\Sigma}^{\varepsilon}$ on $\partial \mathcal{D}$. We form the domain $\mathcal{D}^{\varepsilon}$ on replacing $\hat{\Sigma}^{\varepsilon}$ by $\Sigma^{\varepsilon}$ and removing the region between the arcs.

For the changes in $p$ and in $A$ for small $\varepsilon$, we find up to terms of smaller order in $\varepsilon$

$$
\delta p \approx-\frac{(2 \varepsilon)^{3 / 2}}{3 \sqrt{\kappa-\kappa^{\varepsilon}}}\left(\kappa+\kappa^{\varepsilon}\right), \quad \delta A \approx-\frac{(2 \varepsilon)^{3 / 2}}{3 \sqrt{\kappa-\kappa^{\varepsilon}}} \cdot 2 .
$$

Thus for small $\varepsilon$

$$
\delta\left(\frac{p}{A}\right)=\frac{1}{A}\left\{\delta p-\left(\frac{p}{A}\right) \delta A\right\} \approx \frac{(2 \varepsilon)^{3 / 2}}{3 A \sqrt{\kappa-\kappa^{\varepsilon}}}\left\{-\left(\kappa+\kappa^{\varepsilon}\right)+2 \frac{p}{A}\right\} .
$$

By hypothesis, we may choose $\kappa^{\varepsilon}<\kappa$ and large enough that $-\left(\kappa+\kappa^{\varepsilon}\right)+$ $2 p / A<0$; the assertion then follows on choosing $\varepsilon$ small enough.

If $q$ is the vertex of a protruding corner, we may cut off a tip of the corner with a straight segment orthogonal to the angle bisector at $q$. The assertion then follows by an analogous, somewhat simpler reasoning.

Corollary 3.1. Under the hypotheses of Theorem 3, suppose that for some particular value of $\gamma>0$ there exists a variational solution of (4), (2) in $\mathcal{D}$. Suppose further that $\alpha+\gamma>\pi / 2$ at each vertex point. Then the construction of the theorem can be so effected that a variational solution of (4), (2) exists in $\mathcal{D}^{\varepsilon}$, and thus, for $B$ small enough, $\mathcal{D}$ will lift liquid to a higher level over the entire section $\mathcal{D}^{\varepsilon}$ than does $\mathcal{D}^{\varepsilon}$ itself. 
Proof. Assume for contradiction that a sequence of constructions as indicated, with $\varepsilon \rightarrow 0$, could be found, for which no such variational solution exists. By Property A above, for each $\varepsilon$ in the sequence, there would exist a subdomain $\mathcal{D}^{*}(\varepsilon)$ as indicated, for which $\Phi\left(\mathcal{D}^{*}(\varepsilon) ; \gamma\right) \leq 0$. The radius of each of the subarcs of semicircles $\Gamma^{*}(\varepsilon)$ that bound $\mathcal{D}^{*}(\varepsilon)$ within $\mathcal{D}(\varepsilon)$ is $A^{\varepsilon} / 2 p^{\varepsilon} \cos \gamma$ which remains bounded from zero as $\varepsilon \rightarrow 0$. Since $\gamma>0$, since each arc $\Gamma^{*}(\varepsilon)$ must start and end at a boundary point, since the angle between $\Sigma^{\varepsilon}$ and $\Sigma$ tends to zero, and since $\alpha+\gamma>\pi / 2$ at each vertex, each $\Gamma^{*}(\varepsilon)$ must subtend a length bounded from zero on $\partial \mathcal{D}^{\varepsilon}$. Thus, there can be at most a fixed finite number $N$ of such arcs, for all $\varepsilon$ in the sequence. A subsequence exists, for which all the arcs $\Gamma^{*}$ converge in position and in radius. Since for each $\mathcal{D}^{*}(\varepsilon)$ there holds $\Phi\left(\mathcal{D}^{*} ; \gamma\right) \leq 0$, the same inequality must hold in the limit configuration. It thus remains only to show that the limit configuration is not degenerate, that is, that $\lim \mathcal{D}^{*}(\varepsilon) \neq \varnothing, \mathcal{D}$. Degeneracy can occur only if all the arcs move into an arbitrarily small neighborhood of $\partial \mathcal{D}$. That cannot happen, for the reasons just given.

We note that Theorem 3 gives no information when the outer domain is a disk, as in that case there holds $\kappa(q)=p /(2 A)<p / A$ at all boundary points. In fact, a disk yields a positive answer to Miranda's question relative to any piecewise smooth subdomain (Theorem 5.8 in [4]).

We turn now to further conditions for a positive answer. Siegel [13] provided two kinds of particular conditions under which the answer is positive relative to an inner disk; one of these is especially adapted to our needs. Preliminary to stating the theorem, we observe that for any rotationally symmetric solution $u(r)$ of (3) in a disk centered at the origin there holds

$$
r \frac{u_{r}}{\sqrt{1+u_{r}^{2}}} \equiv r \sin \psi=B \int_{0}^{r} \rho u(\rho) d \rho
$$

where $\psi(r)$ is the inclination angle of the profile curve $u(r)$ with the positively directed $r$-axis. From (16) we see that the $\operatorname{sign}$ of $\sin \psi$ is that of $u(0)$, and thus under our hypothesis $0 \leq \gamma<\pi / 2$ there holds $u(0)>0$. We see that $u(r)$ is increasing, and thus for $r>0$ and within the range of existence we have

$$
\frac{u(0)}{2}<\frac{\sin \psi(r)}{B r}<\frac{u(r)}{2} .
$$

From (17) we conclude the existence of a maximal disk $\hat{\mathcal{D}}_{0}$ of radius $\hat{R}_{0}<$ $2 / B u(0)$ in which such a solution can be defined; there holds $\sin \psi(r) \rightarrow 1$ $\left(u^{\prime}(r) \rightarrow \infty\right)$ as $r \rightarrow \hat{R}_{0}$.

Let $\mathcal{D}_{0}$ be a disk of radius $R_{0}>0, u_{0}(x)$ a solution of (3) in $\mathcal{D}_{0}$ under the boundary condition (2) on $\Sigma_{0}=\partial \mathcal{D}_{0}$. We observe first that $u_{0}(x)$ is radial, since a radial solution exists as is proved in [9], and the solution is unique by Theorem 5.1 of [4]. With some abuse of notation, we denote this solution by 
$u_{0}(r)$. By the above remarks $u_{0}(r)$ can be extended to a maximal domain $\hat{\mathcal{D}}_{0}$ of radius $\hat{R}_{0} \geq R_{0}$. The following result is a slightly sharpened version of the corresponding statement in [13] Theorem 20. The proof we give differs from that in $[\mathbf{1 3}]$ and brings out in somewhat more detail the behavior of the solutions.

Theorem 4. Let $\mathcal{D}_{0}$ be a disk, $u_{0}(r)$ the solution of $(3)$ in $\mathcal{D}_{0}$ under the boundary condition $(2)$ on $\Sigma_{0}=\partial \mathcal{D}_{0}$. Let $\hat{\mathcal{D}}_{0}$ be the maximal domain for this solution (see above). Let $\mathcal{D}_{1}$ be any domain that is piecewise of class $\mathcal{C}^{1}$, such that $\mathcal{D}_{1} \cap \hat{\mathcal{D}}_{0}$ is star-shaped relative to the center of $\mathcal{D}_{0}$ and such that the tangent line at any smooth point of its boundary does not enter $\mathcal{D}_{0}$. Let $u_{1}(x)$ satisfy (3) in $\mathcal{D}_{1} \cap \hat{\mathcal{D}}_{0}$ and (2) at all smooth points of $\hat{\Sigma}_{1}=\partial\left(\mathcal{D}_{1} \cap \hat{\mathcal{D}}_{0}\right) \cap \partial \mathcal{D}_{1}$. Then $u_{0}>u_{1}$ in $\mathcal{D}_{1} \cap \hat{\mathcal{D}}_{0}$, or else $\mathcal{D}_{1}=\hat{\mathcal{D}}_{0}$ and $u_{1} \equiv u_{0}$.

In this theorem, note that the shape of $\partial \mathcal{D}_{1}$ at points exterior to $\hat{\mathcal{D}}_{0}$, and the boundary data achieved by $u_{1}(x)$ at such points, are irrelevant to the result.

Proof of Theorem 4. Let $R_{0}$ be the radius of $\mathcal{D}_{0}$, let $U_{0}(r)$ be the height of a lower spherical cap over $\mathcal{D}_{0}$, satisfying (2) on $\Sigma_{0}$. The cap is unique up to an additive constant, and it extends to a lower hemisphere over a disk $\mathcal{D}_{0}^{+}$ of radius $R_{0}^{+} \geq R_{0}$.

Lemma 4.1. $\mathcal{D}_{0}^{+} \supseteq \hat{\mathcal{D}}_{0}$.

Proof. Let $\varphi$ be the inclination angle of the profile curve $U_{0}(r)$. At $r=$ $R_{0}$, we have by construction $\psi=\varphi$. Thus, the latitudinal curvature $k_{l}=$ $(\sin \psi) / r$ of the surface $u_{0}(r)$ equals at that point the latitudinal curvature $\kappa_{l}$ of the surface $U_{0}(r)$. But $U_{0}(r)$ represents a sphere, so that $\kappa_{l}$ equals the meridional curvature $\kappa_{m}$, and both quantities are independent of $r$. With regard to $u_{0}(r)$, we may write from (16)

$$
k_{l}+k_{m} \equiv \frac{\sin \psi}{r}+(\sin \psi)_{r}=B u_{0}(r) .
$$

There follows from (17) and from (18) that $k_{m}(r) \equiv(\sin \psi)_{r}>k_{l}(r)$ on the entire trajectory, and thus, since $k_{l}\left(R_{0}\right)=\kappa_{l}$, we have $k_{m}\left(R_{0}\right)>\kappa_{l}=\kappa_{m}=$ $(\sin \varphi)_{r}$. From $k_{l}=(\sin \psi) / r$ and $k_{m}=(\sin \psi)_{r}$ we have by $(16)$

$$
k_{l}^{\prime}=B \frac{u_{0}(r)}{r}-2 \frac{\sin \psi}{r^{2}}>0
$$

by (17), and thus

$$
k_{m}(r) \equiv(\sin \psi)_{r}>(\sin \varphi)_{r} \equiv \kappa_{m}
$$

for $r \geq R_{0}$. Since $\sin \psi\left(R_{0}\right)=\sin \varphi\left(R_{0}\right)$, the lemma follows.

We return to the proof of the theorem. Since a plane cuts a sphere in a constant angle, we see that at any point $p$ interior to a smooth interval 
of $\Sigma_{1}$ and also interior to $\hat{\mathcal{D}}_{0}$, we have $\left.\left.\nu \cdot T U_{0}\right]_{p}=\nu \cdot T U_{0}\right]_{p_{\perp}}$, where $p_{\perp}$ is the point on the line tangent to $\Sigma_{1}$ at $p$, that is closest to the center point $p_{0}$ of $\mathcal{D}_{0}$. Since this line by hypothesis does not enter $\mathcal{D}_{0}$, there holds $\left|p_{\perp}-p_{0}\right| \geq R_{0}$, and hence $\left|\nu \cdot T U_{0}\right|_{p} \geq \cos \gamma$. Since $\mathcal{D}_{1} \cap \hat{\mathcal{D}}_{0}$ is star shaped relative to $p_{0}$, the exterior normal on $\Sigma_{1}$ is inclined less than $90^{0}$ from the outwardly directed radial line from $p_{0}$; thus $\nu \cdot T U_{0}>0$ at $p$ and hence $\nu \cdot T U_{0} \geq \cos \gamma$. Since as we have shown, $\sin \psi(r)>\sin \varphi(r)$ at such points, we have also $\nu \cdot T u_{0} \geq \cos \gamma$ at $p$. If there are points of $\hat{\Sigma}_{0}=\partial \hat{\mathcal{D}}_{0}$ that are interior to $\mathcal{D}_{1}$, there would hold $\nu \cdot T u_{0}=1>\nu \cdot T u_{1}$ at such points, since $|\nu \cdot T u|<1$ for any differentiable function $u(x)$. Thus, at all smooth points on the boundary of $\mathcal{D}_{1} \cap \hat{\mathcal{D}}_{0}$, we have $\nu \cdot T u_{0} \geq \nu \cdot T u_{1}$. The remaining boundary points of this domain form a set of linear Hausdorff measure zero. By the general maximum principle Theorem 5.1 of [4], we obtain $u_{0}(x)>u_{1}(x)$ or else the two functions coincide, as was to be shown.

We note that in Theorem 4 there is no restriction on the magnitude of $B$, nor is it necessary to assume existence of a zero gravity solution.

3.

The following example illustrates both Theorem 3 and Theorem 4, and exhibits a striking discontinuity in behavior:

Example. Let $\mathcal{D}_{1}$ be a square of side length 2. We determine an interior "disq domain" $\mathcal{D}_{t}=\mathcal{D}(t)$ by rounding the corners of $\mathcal{D}_{1}$ by circular arcs of radius $(1-t), 0<t<1$. Denoting the perimeter of $\mathcal{D}(t)$ by $p(t)$ and its area by $A(t)$, we find

$$
\frac{p(t)}{A(t)}=2 \lambda(t)=\lambda(t) \frac{p_{1}}{A_{1}}
$$

with

$$
\lambda(t)=\frac{\pi+t(4-\pi)}{\pi+t(2-t)(4-\pi)}<1
$$

for any $t$ in the indicated range.

We wish to apply Corollary 2.1 in order to translate this result into a property of solutions of (3), (2). To that purpose, one needs existence of variational solutions of (4), (2) in $\mathcal{D}(t)$ and in $\mathcal{D}_{1}$. If $\gamma<\pi / 4$, then there is no variational solution of $(4),(2)$ in $\mathcal{D}_{1}$, see the remarks following "Property A" above. But if $\gamma \geq \pi / 4$ then a strict solution of (4) in $\mathcal{D}_{1}$, with data (2) assumed strictly except at the corner points, is available explicitly as a lower spherical cap. This solution is also a variational solution of the problem. The existence of variational solutions in the smoothed domains was proved by Concus and Finn in [2], as a consequence of Property A. Each such solution 
is bounded in its domain, in view of Property B. Thus, for each of the disq domains $\mathcal{D}(t)$, if the gravity field is small enough, a capillary tube with the original square section containing $\mathcal{D}(t)$ will raise liquid to a higher level over all of $\mathcal{D}(t)$ than will $\mathcal{D}(t)$ itself. In fact, for each fixed $t$ the difference of heights will tend to infinity by an amount asymptotically $\lambda / B$, as indicated in Theorem 2.

We may contrast that result with the behavior of solutions in the inscribed disk $\mathcal{D}_{0}$. This disk is smoothly contained in the above family $\mathcal{D}(t)$, from which it is obtained by setting $t=0$. But in this limiting case, one finds $p_{0} / A_{0}=p_{1} / A_{1}$, so that Corollary 2.1 is inconclusive. In fact, by Theorem 4 we see that instead of the solutions in $\mathcal{D}_{0}$ being dominated by those of $\mathcal{D}_{1}$ for small $B$, the inequality goes the other way around, and in fact that happens for all $B>0$. The height $u_{0}$ in the inscribed disk $\mathcal{D}_{0}$ in the example above exceeds at each point of $\mathcal{D}_{0}$ the height for the square and also the height $u_{t}(x ; B)$ in any of the intermediate "disq" domains $\mathcal{D}_{t}$ obtained by smoothing of the corners. There is thus a positive answer to Miranda's question, for any $B>0$, when the inner domain is a disk and the outer domain is either a circumscribed square or any of the disqs indicated above. This is despite the fact that for any of the disq domains, no matter how close to $\mathcal{D}_{0}$, an (arbitrarily large) negative answer occurs between that domain and the square for all small enough $B$.

Even though for each fixed $t \in(0,1)$ there holds $\lim _{B \rightarrow 0}\left(u_{1}(x ; B)-\right.$ $\left.u_{t}(x ; B)\right)=+\infty$, there nevertheless holds $u_{1}(x ; B)-u_{0}(x ; B)<0$ for every $B>0$. This behavior is the more remarkable, as it is easily shown that $\lim _{t \rightarrow 0} u_{t}(x ; B)=u_{0}(x ; B)$, uniformly over compact subsets of $\mathcal{D}_{0}$, for every fixed $B$. For convenience of the reader, we offer a proof of that property in the Appendix. One finds (see $(24)$ below) $\lim _{B \rightarrow 0}\left(u_{1}(x ; B)-u_{0}(x ; B)\right) \equiv$ const. $=-\Lambda$. Although $u_{t}(x ; B)$ is continuous in $t$ at $t=0$ for each fixed $B>0$, its limiting behavior as $B \rightarrow 0$ changes discontinuously in $t$ at that value.

The following remarks may lend further insight to the situation. We consider a representation for solutions of (3), (2) proved by D. Siegel [14] (see also Miersemann [12]) under the hypothesis that the domain $\mathcal{D} \in C^{2+\alpha}$. Although each $D(t)$ in the above example contains eight boundary points with discontinuous second derivatives, and $\mathcal{D}_{1}$ has four boundary points with discontinuous normal, we will presume that the result of Siegel can be applied. Siegel assumes the existence of a strict solution $U(x)$ of (4), (2) in a domain $\mathcal{D}$, and normalizes it by an additive constant to have mean value zero over $\mathcal{D}$. He then proves that any solution $u(x ; B)$ of $(3),(2)$ over $\mathcal{D}$ has the asymptotic behavior

$$
u(x ; B)=\frac{p}{B A} \cos \gamma+U(x)+O(B) .
$$


The solutions $U_{0}(x)$ and $U_{1}(x)$ over the inscribed disk and the square are known explicitly as lower spherical caps of the same radius, and thus differ by a constant. A formal calculation yields

$$
\begin{aligned}
& \frac{1}{R}\left(U_{0}(x)-U_{1}(x)\right) \\
& =\frac{1}{12 \cos ^{3} \gamma}\left\{2 \tan ^{-1} \frac{-1+\cos \gamma+\cos ^{2} \gamma}{\cos \gamma \sqrt{1-2 \cos ^{2} \gamma}}-2 \tan ^{-1} \frac{-1-\cos \gamma+\cos ^{2} \gamma}{\cos \gamma \sqrt{1-2 \cos ^{2} \gamma}}\right. \\
& \quad+\left(\cos ^{3} \gamma-9 \cos \gamma\right) \sin ^{-1} \cot \gamma-4 \cos ^{2} \gamma \sqrt{1-2 \cos ^{2} \gamma} \\
& \left.\quad-3 \cos \gamma \sin ^{2} \gamma \tan ^{-1} \frac{\cos \gamma}{\sqrt{1-2 \cos ^{2} \gamma}}+8-8 \sin ^{3} \gamma\right\} \\
& =\Lambda(\gamma)>0 .
\end{aligned}
$$

Since the values $p / A$ are the same for $\mathcal{D}_{0}$ and $\mathcal{D}_{1}$, we find

$$
u_{0}(x ; B)-u_{1}(x ; B)=U_{0}(x)-U_{1}(x)+O(B) \equiv \Lambda(\gamma)+O(B)
$$

as $B \rightarrow 0$. This relation provides an asymptotically precise formulation of our qualitative result above based on Theorem 4 .

The intermediate domains $\mathcal{D}(t)$ exhibit a very different dependence on $B$. We find in fact by Siegel's representation

$$
\begin{aligned}
& u_{1}(x ; B)-u_{t}(x ; B)=\frac{1}{B}\left(\frac{2}{R}-\frac{p_{t}}{A_{t}}\right) \cos \gamma+U_{1}(x)-U_{t}(x)+O(B) \\
& u_{0}(x ; B)-u_{t}(x ; B)=\frac{1}{B}\left(\frac{2}{R}-\frac{p_{t}}{A_{t}}\right) \cos \gamma+U_{0}(x)-U_{t}(x)+O(B)
\end{aligned}
$$

for each fixed $t \in(0,1)$; this again brings out the close relationship between the solutions in $\mathcal{D}_{0}$ and $\mathcal{D}_{1}$, as opposed to those in the intermediate domains $\mathcal{D}(t)$, since the quantity in brackets is positive and the same for both expressions. Thus, each of the differences on the left side of (26) becomes arbitrarily large for decreasing $B$.

From another point of view, we have

$$
\lim _{t \rightarrow 0}\left\{u_{1}(x ; B)-u_{t}(x ; B)\right\}=u_{1}(x ; B)-u_{0}(x ; B) .
$$

By (21), (22) and Corollary 2.1, the quantity in brackets in (27) is positive at each $t$ for all sufficiently small $B$. But the right side of (27) is negative for every $B>0$, by Theorem 4 . Thus there can be no uniform choice of $B$ for which $u_{1}(x ; B)-u_{t}(x ; B)$ stays positive for all $t \in(0,1)$. Beyond that, we have

$$
\lim _{B \rightarrow 0} \lim _{t \rightarrow 0}\left\{u_{1}(x ; B)-u_{t}(x ; B)\right\}=U_{1}(x)-U_{0}(x)=-\Lambda
$$


by (27), (25), (24), while

$$
\lim _{B \rightarrow 0}\left\{u_{1}(x ; B)-u_{t}(x ; B)\right\}=\infty
$$

so the limiting order cannot be interchanged.

The behavior described in the example above was verified by formal computer calculations of the surfaces. This work will appear elsewhere in an independent paper, currently in preparation, by V. Brady, P. Concus, and R. Finn.

\section{4.}

The above result expressed in Theorem 4 , that within $\mathcal{D}_{0}$ the inscribed disk solution $u_{0}(x ; B)$ lies above any of the disq solutions, is put into further relief by the observation that given any domain $\hat{\mathcal{D}}_{0}$ with area equal to that of $\mathcal{D}_{0}$ and which admits a variational solution of (4), (2), then $\sup _{\mathcal{D}_{0}} u_{0}(x ; B)<\inf _{\hat{\mathcal{D}}_{0}} \hat{u}(x ; B)$ for all small enough $B$. That follows immediately from Theorem 2 and from the isoperimetric inequality. Thus, any modification of one of the domains $\mathcal{D}(t)$ for any $t \in(0,1)$ that decreases its area to that of $\mathcal{D}_{0}$ and preserves the existence property for (4), (2), will reverse the conclusion in the second paragraph following (22). Such a modification can be effected, e.g., by a similarity transformation. The change is the more remarkable, in that all height differences become unboundedly large for decreasing $B$.

\section{5.}

The properties of the disq construction suggest that the result of Theorem 3 cannot be substantively improved. In fact, as $t$ decreases from 1 to 0 the values $p / A$ at first decrease monotonically from 2 to a minimum $(p / A)_{\min }=$ $1+\sqrt{\pi} / 2$, at a value $t_{m}=\sqrt{\pi} /(2+\sqrt{\pi})$. In this interval, the curvature $\kappa$ of the smoothing arc satisfies $\kappa>p / A$, and indeed for such arcs the Miranda question has a negative answer for all the disq domains in the remaining range to the minimum point $t=t_{m}$. At $t=t_{m}$ there holds exactly $\kappa=p / A$, and beyond that point one finds $\kappa<p / A$, until $\kappa=p / 2 A$ for the inscribed disk. The domains of the smoothing family corresponding to values $t<t_{m}$ yield heights that are above those produced by the configuration for which $t=t_{m}$. The change in behavior occurs exactly at the curvature value that appears in the statement of Theorem 3.

Under the hypotheses of Theorem 3 , suppose in addition that $\mathcal{D}$ is starshaped relative to $x_{0} \in \mathcal{D}$ and that no tangent to a smooth point of $\partial \mathcal{D}$ enters a disk $\mathcal{D}_{0}$ centered at $x_{0}$. For prescribed data $\gamma$ and $B>0$, let $u(x ; B)$ and $u_{0}(x ; B)$ be solutions of $(3),(2)$ in $\mathcal{D}$ and in $\mathcal{D}_{0}$. Then by Theorem 4 , $u_{0}(x ; B)>u(x ; B)$ in $\mathcal{D}_{0}$, and more generally in the extended domain $\hat{\mathcal{D}}_{0} \cap \mathcal{D}$. 
Thus, the situation is formally analogous to that of the inscribed disk in the square described above. Both $u(x ; B)$ and $u_{0}(x ; B)$ dominate the solution determined by the intermediate domain $\mathcal{D}^{\varepsilon}$ when $B$ is sufficiently small; in $\hat{\mathcal{D}}_{0} \cap \mathcal{D}^{*}, u_{0}(x ; B)$ dominates the solution in any domain $\mathcal{D}^{*}$ for every $B>0$, whenever $\partial \mathcal{D}^{*}$ satisfies the geometrical requirement.

Given any domain $\mathcal{D}$, a local deformation at the boundary will create a new domain to which the criterion of Theorem 3 can be applied. The following results provide partial information as to what can be expected in general, and have also an independent interest. We prove first:

Theorem 5. Let $\mathcal{D} \in \mathcal{C}^{1}$ be convex, with area $A$ and perimeter $p$. Let $\Omega_{0}$ be a disk of maximal radius $r_{0}$ inscribed in $\mathcal{D}$. Then $r_{0} \leq 2 A / p$, equality holding only if $\mathcal{D}$ coincides with $\Omega_{0}$. If $\mathcal{D}$ is known only to be convex, then $r_{0} \leq 2 A / p$, equality holding only if $\partial \mathcal{D}$ consists of a countable number of arcs of $\partial \Omega_{0}$ joined with countably many supporting lines of $\Omega_{0}$. Conversely, if $\partial \mathcal{D}$ has that form, then $r_{0}=2 A / p$.

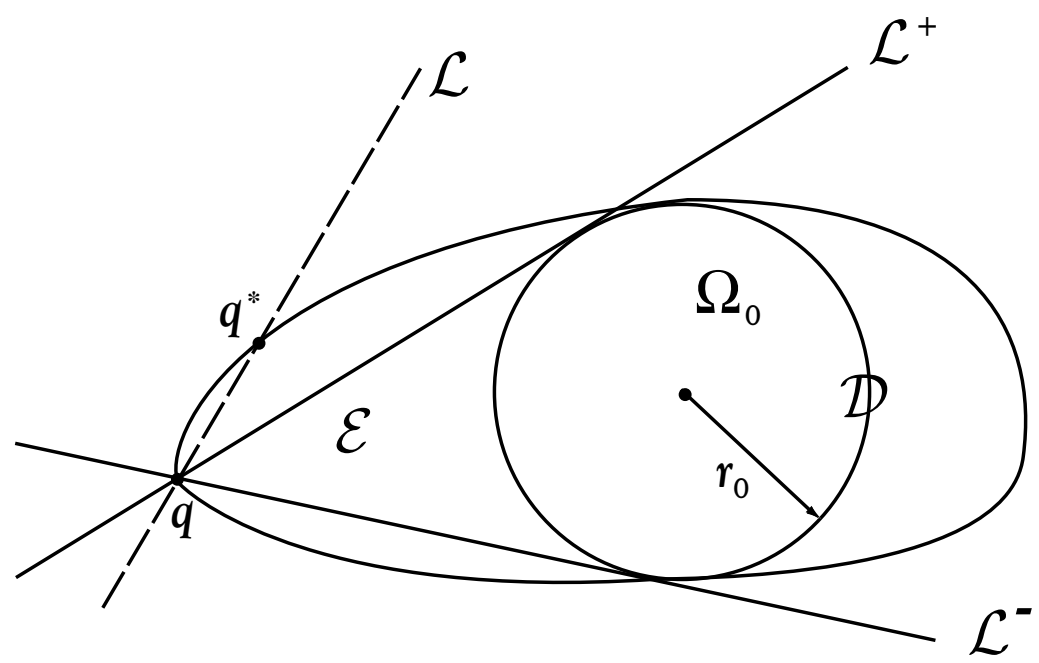

Figure 2a. Proof of Theorem 5; initial steps.

Proof. We approximate $\partial \mathcal{D}$ by a convex circumscribed polygon of $n$ sides. Denoting by $h_{j}$ the distance from the center $O$ of $\Omega_{0}$ to the (linearly extended) $j$-th segment, and by $\delta l$ the length of that segment, the $n$-th approximation to the area yields

$$
A_{n}=\frac{1}{2} \sum_{1}^{n} h_{j} \delta l_{j} \geq \frac{1}{2} r_{n} \sum_{1}^{n} \delta l_{j}
$$


where $r_{n}$ is the smallest of the $h_{j}$. Since the linear extension of each segment lies exterior to $\Omega_{0}$, we have $\lim _{n \rightarrow \infty} r_{n}=r_{0}$, and thus $A=\lim _{n \rightarrow \infty} A_{n} \geq$ $\frac{1}{2} r_{0} p$, as asserted. If $\mathcal{D}$ and $\Omega_{0}$ do not coincide, let $q$ denote a boundary point of $\mathcal{D}$ that is exterior to $\Omega_{0}$ and of maximal distance from $O$, and denote by $\mathcal{L}^{-}, \mathcal{L}^{+}$the support lines of $\Omega_{0}$ containing $q$. Referring to Figure $2 \mathrm{a}$, we note first that no points of the domain $\mathcal{E}$ cut off by these lines can be boundary points of $\mathcal{D}$, as otherwise there would be a segment containing three boundary points and also interior points, which is excluded by the convexity of $\mathcal{D}$. Suppose there were a boundary point $q^{*} \notin \mathcal{L}^{+}$on the side of $\mathcal{L}^{+}$exterior to $\Omega_{0}$. Then the segment joining $q$ to $q^{*}$ would lie on a line $\mathcal{L}$ situated exterior to $\Omega_{0}$, and every point $\tau$ on the segment of $\mathcal{L}$ between $q$ and $q^{*}$ would lie on a distinct line $\mathcal{L}_{\tau}$ through $O$. Since every convex domain is star shaped with respect to each of its points, the points $\tau$ biuniquely correspond to intersection points of $\mathcal{L}_{\tau}$ with $\partial \mathcal{D}$. We introduce the support line $\mathcal{L}^{*}$ of $\Omega_{0}$ through $q^{*}$ as in Figure $2 \mathrm{~b}$, and the point $q^{* *}$ determined by the intersection of $\mathcal{L}^{*}$ with a concentric circle through $q$, as indicated in the figure. We observe that at all interior points of the segment

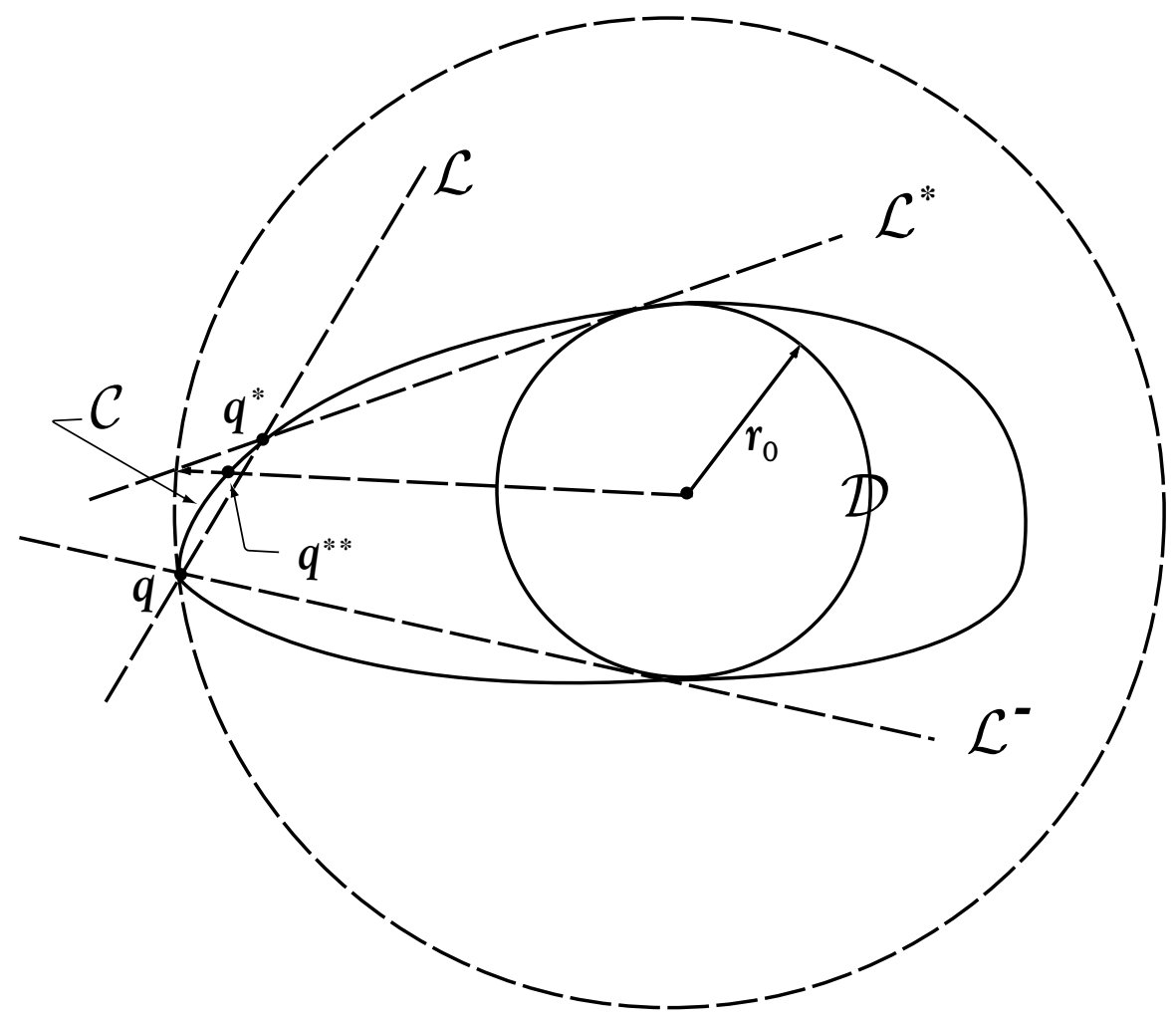

Figure 2b. Proof of Theorem 5; concluding steps. 
$\mathcal{C}$ of $\partial \mathcal{D}$ between $q^{*}$ and $q^{* *}$, support lines of $\mathcal{C}$ cannot cross that segment and thus all such lines lie exterior to $\Omega_{0}$. On any compact subset of $\mathcal{C}$ all support lines are bounded from $\Omega_{0}$. Since $\mathcal{C}$ has positive length, there would hold $A=\lim _{n \rightarrow \infty} A_{n}>\frac{1}{2} r_{0} p$. We conclude, since $\partial \mathcal{D}$ is connected, that if $r_{0}=2 A / p$ then the two support segments from $q$ to $\Omega_{0}$ are contained in $\partial \mathcal{D}$, and that they are support segments of $\mathcal{D}$.

We next repeat the procedure, restricting attention to the complement of these segments. Continuing in that way, we establish the asserted form of $\mathcal{D}$, in an at most countable number of steps.

The final assertion of the theorem, that every such domain satisfies $r_{0}=$ $2 A / p$, is established by formal evaluation of the area and perimeter of the figure.

We may now prove:

Theorem 6. Let $\mathcal{D} \in \mathcal{C}^{2}$ be convex, and denote by $\kappa$ its boundary curvature. Then either $\mathcal{D}$ is a circular disk or else there exists $q \in \partial \mathcal{D}$ at which $\kappa(q)>$ $p / 2 A$.

Strict convexity is not needed for the result; however, convexity (or something in that direction) is essential, as one sees from Figure 3.

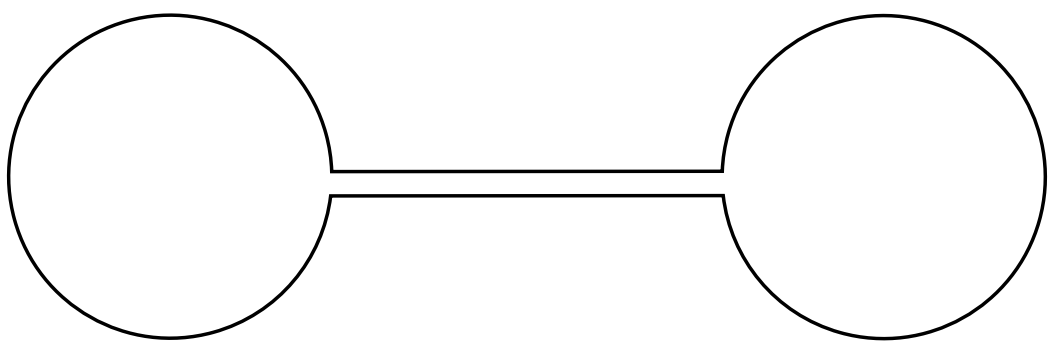

Figure 3. Convexity is needed for Theorem 6 .

Proof. Let $\Omega_{0}$ be a disk of maximal radius $r_{0}$ inscribed in $\mathcal{D}$, let $\kappa_{0}=1 / r_{0}$. We observe first that any semicircle on $\partial \Omega_{0}$ must contain at least one contact point with $\mathcal{D}$ : Otherwise it would be possible to move $\mathcal{D}_{0}$ into $\mathcal{D}$ and then increase its radius within $\mathcal{D}$, contrary to the construction. If $\Omega_{0}$ is not identically $\mathcal{D}$ then there would be a point $q \in \partial \Omega_{0}$, with $q$ interior to $\mathcal{D}$. By the above remark, the largest segment on $\partial \Omega_{0}$ that contains $q$ and contains no points of $\partial \mathcal{D}$ cannot exceed a semicircle. We thus obtain a configuration as in Figure 4, with an arc of $\partial \Omega_{0}$ and an arc of $\partial \mathcal{D}$ both passing through the two endpoints of a segment, both having equal slopes at the endpoints, and both expressible as graphs over the segment. Denoting the angle between the tangent to $\partial \Omega_{0}$ and the direction of the segment by $\varphi$ and the corresponding 


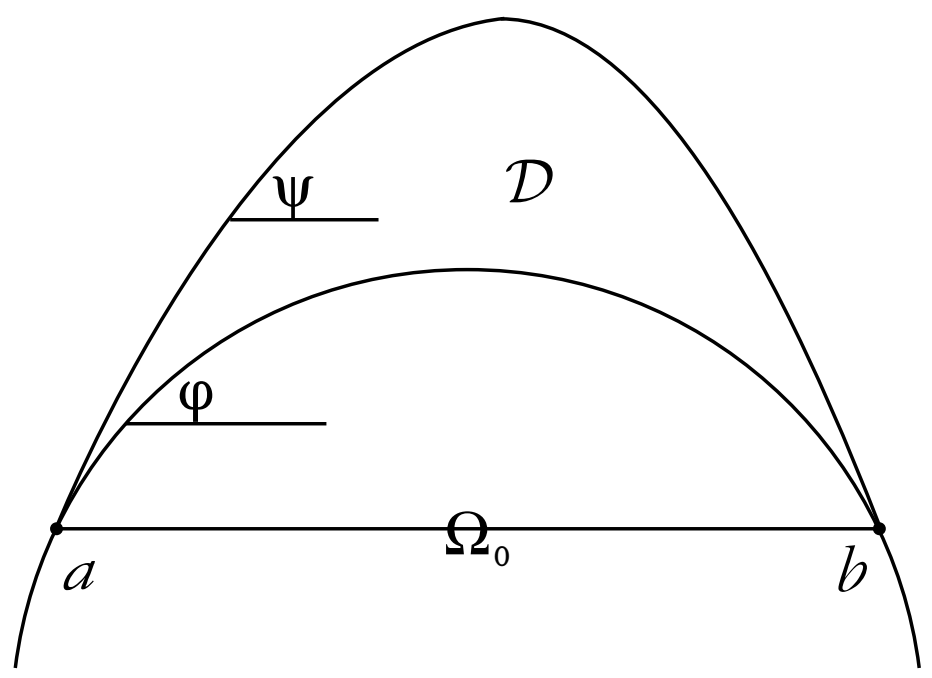

Figure 4. Proof of Theorem 6 .

angle on $\partial \mathcal{D}$ by $\psi$, we have $(-\sin \varphi)_{x}=\kappa_{0}>p / 2 A$ by Theorem 5 , while $(-\sin \psi)_{x}=\kappa$, the curvature on $\partial \mathcal{D}$. Thus,

$$
\int_{a}^{b}\left(\kappa-\kappa_{0}\right) d x=0
$$

and we conclude that either $\kappa \equiv \kappa_{0}$ on the segment, or else there exist points where $\kappa>\kappa_{0}$. The theorem follows.

Returning to the Miranda question, we have:

Theorem 7. Let $\mathcal{D}_{0}, \mathcal{D}_{1}$ be piecewise of class $\mathcal{C}^{1}$, let $\mathcal{D}_{0} \subset \mathcal{D}_{1}$ and suppose that $\mathcal{D}_{1}$ admits a variational solution of (4), (2). Suppose that $\mathcal{D}_{0}$ lies interior to a disk $\mathcal{B}$ of radius $R<2 A_{1} / p_{1}$, where $A_{1}, p_{1}$ are the area and perimeter of $\mathcal{D}_{1}$. Let $u_{0}(x ; B), u_{1}(x ; B)$ be variational solutions of $(3),(2)$ in $\mathcal{D}_{0}$ and in $\mathcal{D}_{1}$. Then

$$
\lim _{B \rightarrow 0}\left\{\inf _{\mathcal{D}_{0}} u_{0}(x ; B)-\sup _{\mathcal{D}_{0}} u_{1}(x ; B)\right\}=+\infty .
$$

Proof. Denote by $w(x ; B)$ the solution of $(3),(2)$ in $\mathcal{B}$. By Theorem 5.8 of [4], there holds $u_{0}(x ; B)>w(x ; B)$ throughout $\mathcal{D}_{0}$. A solution of $(4),(2)$ in $\mathcal{B}$ is known explicitly as a spherical cap. By Theorem 1 above, there holds

$$
\left|w(x ; B)-\frac{2}{B R} \cos \gamma\right|<C<\infty
$$


for all $B>0$. By hypothesis, we may write $R=2 \lambda A_{1} / p_{1}$ with $0<\lambda<1$; hence by $(33)$

$$
w(x ; B)>\frac{p_{1}}{\lambda B A_{1}} \cos \gamma-C .
$$

But by Theorem 1 we have for all $B>0$ that

$$
\left|u_{1}(x ; B)-\frac{p_{1}}{B A_{1}} \cos \gamma\right|<C_{1}<\infty
$$

and the result follows.

In interpreting Theorem 7, we note the related result:

Theorem 8. Let $\mathcal{D}_{1} \in \mathcal{C}^{1}$ be convex and $\Omega_{0}$ the maximal inscribed disk. Suppose that $\mathcal{D}_{0} \subset \Omega_{0}$ and that $\mathcal{D}_{0}, \mathcal{D}_{1}$ admit variational solutions of (4), (2). Then either all three domains are identical, or else (32) holds.

The proof is immediate from Theorem 5 above, and from the isoperimetric inequality applied to $\mathcal{D}_{0}$.

We close with a conjecture:

Conjecture. Suppose the boundary curvature $\kappa$ of a convex domain $\mathcal{D}$ satisfies $\kappa<p / A$ at all points. Then for any contact angle $\gamma$ in $0 \leq \gamma<\pi / 2$ and any downward directed gravity field, any strict subdomain $\mathcal{D}_{0} \subset \mathcal{D}$ will lift fluid higher over all points of its section than will $\mathcal{D}$.

The result of the conjecture would be a generalization of Theorem $5.8 \mathrm{in}$ [4], which provides that result in the particular case for which $\mathcal{D}$ is a disk.

Acknowledgements. We wish to thank Paul Concus and G.N. Krichevets for several helpful discussions. We thank Darren Lee for symbolic calculations with Maple software that yielded the relation (24). This research was supported in part by the National Science Foundation. The former author is indebted to the Max-Planck-Institut für Mathematik in den Naturwissenschaften, in Leipzig, for its hospitality during preparation of the paper.

\section{Appendix.}

We prove here the result stated in Section 3 above, that if $u_{t}(x ; B)$ are variational solutions of (3), (2) in $\mathcal{D}_{t}$ for a fixed $B>0$, there holds $\lim _{t \rightarrow 0} u_{t}(x ; B)$ $=u_{0}(x ; B)$ at each $x \in \mathcal{D}_{0}$. We are given

$$
\int_{\mathcal{D}_{t}}\left(\nabla \eta \cdot T u_{t}-B u_{t} \eta\right) d x-\oint_{\partial \mathcal{D}_{t}} \eta \cos \gamma d s=0
$$

for all $\eta \in \mathcal{Q}\left(\mathcal{D}_{t}\right)$, and

$$
\int_{\mathcal{D}_{0}}\left(\nabla \eta \cdot T u_{0}+B u_{0} \eta\right) d x-\oint_{\partial \mathcal{D}_{0}} \eta \cos \gamma d s=0
$$


for all $\eta \in \mathcal{Q}\left(\mathcal{D}_{0}\right)$.

Let $\eta \in \mathcal{Q}\left(\mathcal{D}_{0}\right)$. Then $\eta\left(\frac{1}{1+(\sqrt{2}-1) t} x\right) \in \mathcal{Q}\left(\mathcal{D}_{t}\right)$. For this $\eta$, we find

$$
\begin{aligned}
& \int_{\mathcal{D}_{0}}\left(\nabla \eta \cdot\left(T u_{0}-T u_{t}\right)+B\left(u_{0}-u_{t}\right) \eta\right) d x \\
& -\int_{\mathcal{D}_{t} \backslash \mathcal{D}_{0}}\left(\nabla \eta \cdot T u_{t}+B \eta u_{t}\right) d x-\oint_{\partial \mathcal{D}_{0}} \eta \cos \gamma d s+\oint_{\partial \mathcal{D}_{t}} \eta \cos \gamma d s=0 .
\end{aligned}
$$

The domains $\mathcal{D}_{t}$ satisfy a uniform internal sphere condition (see [7] or [4] Sec. 5.4). It follows that the functions $u_{t}$ are bounded throughout $\mathcal{D}_{t}$, independent of $t$. From general estimates [11], all derivatives of these functions are uniformly bounded in every compact subdomain of $\mathcal{D}_{0}$, and thus there is a subsequence of the $u_{t}$ that converges, uniformly in each such subdomain, to a solution $\hat{u}_{0}(x) \in \mathcal{Q}\left(\mathcal{D}_{0}\right)$ of $(3)$ in $\mathcal{D}_{0}$.

Since $\left|T u_{j}\right|<1$, all $j$, the second integral in (38) vanishes in the limit. Similarly, the last two integrals combine to vanish in the limit. In view of the uniform bound on the $u_{t}$, we obtain

$$
\int_{\mathcal{D}_{0}}\left(\nabla \eta \cdot\left(T u_{0}-T \hat{u}_{0}\right)+B\left(u_{0}-\hat{u}_{0}\right) \eta\right) d x=0 .
$$

The choice $\eta=u_{0}-\hat{u}_{0}$ leads to the conclusion $u_{0} \equiv \hat{u}_{0}$, since $\nabla\left(u_{0}-\hat{u}_{0}\right)$. $\left(T u_{0}-T \hat{u}_{0}\right) \geq 0$ and vanishes only if $\nabla u_{0}=\nabla \hat{u}_{0}$, see, e.g., [4] Sec. 5.1.

\section{References}

[1] P. Concus and R. Finn, On the height of a capillary surface, Math. Zeit., 147 (1976), 93-95, MR 53 \#3841, Zbl 0347.76012.

[2] _ Dichotomous behavior of capillary surfaces in zero gravity, Microgr. Sci. Tech., 3 (1990), 87-92.

[3] M. Emmer, Esistenza, unicità e regolarità nelle superfici di equilibrio nei capillari, Ann. Univ. Ferrara, 18 (1973), 79-94, MR 49 \#1281, Zbl 0275.49005.

[4] R. Finn, Equilibrium Capillary Surfaces, Springer-Verlag, 1986; Russian translation: Mir Publishers, 1988, MR 88f:49001, Zbl 0583.35002.

[5] _ A subsidiary variational problem and existence criteria for capillary surfaces, J. Reine Angew. Math., 353 (1984), 196-214, MR 86h:49046, Zbl 0542.49021.

[6] _ A limiting geometry for capillary surfaces, Ann. Sc. Norm. Sup. Pisa, 11 (1984), 361-379, MR 87f:49051, Zbl 0564.49022.

[7] R. Finn and C. Gerhardt, The internal sphere condition and the capillary problem, Ann. Mat. Pura Appl., 112 (1977), 13-31, MR 55 \#7106, Zbl 0349.49019.

[8] C. Gerhardt, Global regularity of solutions to the capillarity problem, Ann. Sc. Norm. Sup. Pisa, 3 (1976), 157-175, MR 58 \#29199, Zbl 0338.49008.

[9] W.E. Johnson and L.M. Perko, Interior and exterior boundary value problems from the theory of the capillary tube, Arch. Rational Mech. Anal., 29 (1968), 125-143, MR 36 \#6686, Zbl 0162.57002. 
[10] A.A. Kosmodem'yanskii, Jr., The comparison of capillary surface heights in case of small gravity, Nonlinear Analysis, Ser. A, 43 (2001), 937-942, MR 2001m:76021, Zbl 0976.76018.

[11] O.A. Ladyzhenskaya and N.N. Ural'tseva, Local estimates for gradients of solutions of non-uniformly elliptic and parabolic equations, Comm. Pure Appl. Math., 23 (1970), 677-703, MR 42 \#654, Zbl 0193.07202.

[12] E. Miersemann, On the rise height in a capillary tube of general cross section, Asymptotic Analysis, 7 (1993), 301-307, MR 94i:76027, Zbl 0794.35018.

[13] D. Siegel, Height estimates for capillary surfaces, Pacific J. Math., 88 (1980), 471-516, MR 82h:35037, Zbl 0453.35030.

[14] _ The behavior of a capillary surface for small Bond number, in "Variational methods for free surface interfaces," eds. P.Concus, R.Finn (Menlo Park, Calif., 1985), Springer-Verlag, New York-Berlin, (1987), 109-113, MR 88f:53010.

[15] N.N. Ural'tseva, Solvability of the capillary problem, Vestnik Leningrad. Univ. Mat., 19 (1973), 54-64; English translation in Vestn. Leningr. Univ., Math., 6 (1979), 363375, Zbl 0419.35040.

[16] Solvability of the capillary problem II, Vestnik Leningrad. Univ. Mat., 1 (1975) 143-149; English translation in Vestn. Leningr. Univ. Math., 8 (1980), 151158, Zbl 0436.35023.

Received August 15, 2000.

Mathematics Department

STANFORD UNIVERSITY

STANFORD, CA 94305-2125

E-mail address: finn@gauss.stanford.edu

Moscow State University

Dept. of Computational Mathematics

ul. Obraztsova 15

103055 Moscow, Russia

E-mail address: mitja@farber.mccme.ru 